\title{
Effect of drug reminder packaging on medication adherence: a systematic review revealing research gaps
}

\author{
Fabienne Boeni ${ }^{1 *}$, Esther Spinatsch ${ }^{1}$, Katja Suter ${ }^{2}$, Kurt E Hersberger ${ }^{1}$ and Isabelle Arnet ${ }^{1}$
}

\begin{abstract}
Background: This was a systematic review of the literature in accordance with the Preferred Reporting Items for Systematic Reviews and Meta-Analyses (PRISMA) statement. Evidence mapping was used to reveal the effect of drug reminder packaging on medication adherence, to identify research gaps and to make suggestions for future research.
\end{abstract}

Methods: PubMed, Embase, CINAHL and PsycINFO were searched with an end date of September 2013 using the Medical Subject Headings (MeSH) term 'medication adherence' and 20 different search terms for 'drug reminder packaging', limited to the English and German languages. Additional references were identified through cross-referencing. All prospective controlled trials with an intervention using drug reminder packaging for patients taking at least one medication without the assistance of a health-care professional were included in the evidence mapping of the effect of drug reminder packaging on adherence and outcomes according to the Economic, Clinical and Humanistic Outcomes (ECHO) model.

Results: A total of 30 studies met the inclusion criteria: 10 randomized controlled trials, 19 controlled clinical trials and 1 cohort study. Drug reminder packaging had a significant effect on at least one adherence parameter in 17 studies (57\%). The methodological quality was strong in five studies. Two studies provided complete information. Clear research gaps emerged.

Conclusions: Overall, the studies showed a positive effect of drug reminder packaging on adherence and clinical outcomes. However, poor reporting and important gaps like missing humanistic and economic outcomes and neglected safety issues limit the drawing of firm conclusions. Suggestions are made for future research.

Keywords: medication adherence, patient compliance, polypharmacy, drug reminder packaging, multicompartment adherence aid, pillbox, multidrug punch card, blister pouch, dose-dispensing service

\section{Background}

Adherence is defined as the extent to which a patient's behavior matches the agreed recommendations from the prescriber [1]. Reported rates vary from $4.6 \%$ to $100 \%$ of patients of all age classes with different medical conditions and on long- or short-term treatments [1,2]. Mean adherence rates for specific diseases are $88.3 \%$ for HIV infection, $76.6 \%$ for cardiovascular disease, $67.5 \%$ for

\footnotetext{
*Correspondence: fabienne.boeni@unibas.ch

'Pharmaceutical Care Research Group, Department of Pharmaceutical Sciences, University of Basel, Klingelbergstrasse 50, CH-4056 Basel, Switzerland

Full list of author information is available at the end of the article
}

diabetes mellitus and $58 \%$ for psychosis patients [2,3]. Adherence depends on patients' capability (e.g., physical, cognitive and economic) and willingness to initiate and execute their treatment plan: if either is insufficient, unintentional or intentional non-adherence will be the consequence $[4,5]$. Non-adherence is known to impair clinical, economic and humanistic outcomes [6-12]. In a study across five European countries, increasing the percentage of patients adhering to antihypertensive treatment to $70 \%$ was estimated to lead to a reduction of cardiovascular related health-care costs by $€ 332$ million (\$461 million) [13]. Reasons for non-adherence are 
highly individual and complex. Therefore, individual needs and necessities have to be assessed to find the optimal aid for each patient.

Drug reminder packaging, such as weekly pillboxes or multidrug punch cards, is widely used in everyday practice. It usually consists of a certain number of compartments containing solid oral medication for specific dosing times. Compared to other adherence-enhancing programs, such as patient counseling, education or motivation [14], drug reminder packaging is a simple technical option and requires little resources on the patient's as well as on the provider's side. The provision of drug reminder packaging aims at enhancing adherence by facilitating medication organization and intake, by decreasing medication errors and by (self-) monitoring medication intake. Various authors suggest that drug reminder packaging supports mainly unintentionally nonadherent patients, e.g., geriatric patients and patients with complex drug regimens [4,15-17]. Previous reviews with restrictive inclusion criteria investigated the effect of reminder packaging on adherence and were inconclusive [18-20]. This review uses evidence mapping [21,22] to analyze data from a different perspective, highlighting methodological strength and completeness of information as well as research gaps, to identify areas for future research.

\section{Methods}

A systematic review was conducted, complying with the Preferred Reporting Items for Systematic Reviews and Meta-analyses (PRISMA) statement. We proceeded by following the evidence mapping methodology in four steps: question development, question prioritization, evidence search and selection, and data extraction [21].

\section{Question development and prioritization}

The study question was deduced from previous reviews. An evidence report was composed after a preliminary literature search. Keywords were defined based on the results of this search. Experts were consulted to prioritize the question.

\section{Literature search}

PubMed, Embase, CINAHL and PsycINFO were searched for articles published up until September 2013. The keywords used in the search strategy were the Medical Subject Headings $(\mathrm{MeSH})$ term 'medication adherence' and 20 different terms for 'drug reminder packaging': unit dose", reminder pack", unit of use pack*, pill organiser, pill organizer, medication packaging, medication container, pill container, pill box, pillbox, pill calendar, calendar pack", calendar blister pack", doset", dosset", blister pack", pill pack", special packaging AND medication, drug pack", webster pack. The search was restricted to the English and
German languages. Abstracts were screened and full text articles of potential hits were retrieved. References of retrieved articles were screened for relevant crossreferenced articles.

\section{Study selection and data extraction}

The full text of potentially relevant articles was reviewed. Inclusion criteria were any prospective controlled study design, with at least one outcome being adherence, economic, clinical or humanistic, with drug reminder packaging as an intervention in any adherence-enhancing program, for patients taking one or more oral medication (prescribed or over-the-counter) without the help of a health-care professional. Trials were excluded if they were performed in developing countries or if they used drug reminder packaging with incorporated electronic features (e.g., the Medical Event Monitoring System). Drug reminder packaging included reusable multicompartment adherence aids (plastic pillboxes with several compartments per day or per week filled by the patient or pharmacy staff), non-reusable multidrug punch cards (frame cards with plastic cavities, sealed with a foil backing, with typically 28 compartments, filled by pharmacy staff, by a specialized company or an automated system) and non-reusable unit-of-use packaging (e.g., blister pouches attached to form flexible chains, with an unrestricted number of separated daily dosing times, filled by automated systems) [23].

Data extracted included the author, publication year, study design, duration of the intervention and follow-up, description of the participants (e.g., age, clinical conditions and number of medications), outcomes, method of adherence measurement, type of drug reminder packaging and additional interventions. The literature selection and analysis of methodological issues were performed independently by two reviewers. Consensus regarding the results was reached by discussion.

\section{Methodological quality and completeness of information}

The methodological quality of the studies was assessed using the tool for quantitative studies developed for public health topics by the Effective Public Health Practice Project (EPHPP) group [24]. In brief, the tool is applicable to a variety of study designs other than randomized controlled trials (RCTs), such as pre- and post-cohort studies and case-control studies, and it has been validated [25]. It assesses eight components: (1) selection bias, (2) study design, (3) confounders, (4) blinding, (5) data collection method, (6) withdrawals and dropouts, (7) intervention integrity and (8) analysis. Components 1 to 6 were rated as strong, moderate or weak. Based on the rating of the components, studies were described as of weak, moderate or strong methodological quality $[24,26]$. The tool was adapted to the review question. 
The component '(4) blinding' was not assessed because it is not applicable in studies investigating adherence with drug reminder packaging. The rating of criterion '(5) data collection method' focused on adherence outcomes [4]. Data collection was considered 'valid and reliable': (a) if the calculation of the medication possession ratio, the calculation of the medication refill frequency, therapeutic drug monitoring or a validated questionnaire were applied as a single method; (b) if pill count or clinical parameters were combined with at least one additional adherence measurement method (e.g., therapeutic drug monitoring) and (c) if appointment keeping was combined with at least two additional adherence measurement methods.

Following the recommendations of the CONSORT (Consolidated Standards of Reporting Trials) statements for non-pharmacological treatment [27] and the Cochrane Handbook [28], eight additional criteria were selected to assess completeness of information (Table 1). One point was accredited per reported criterion. 'Completeness of information' was defined as the sum of the points divided by eight, resulting in rates from 0 (no item on completeness of information available) to 1 (all items on completeness of information available). The packaging was defined as 'described' if the design (daily, weekly or monthly) and the number of cavities were reported. Criteria 7 and 8, concerning medication not packed in the drug reminder packaging, were not applicable if it was stated that all medication was packed into a drug reminder packaging device. Results were calculated according to the adjusted denominator.

\section{Outcomes}

Any measurement estimating taking adherence (i.e., an indicator of taken medication) was extracted as an adherence outcome. The Economic, Clinical and Humanistic Outcomes (ECHO) model [29] was used to classify further study outcomes. Therapeutic drug monitoring, biomarker and physiological measurements were categorized as

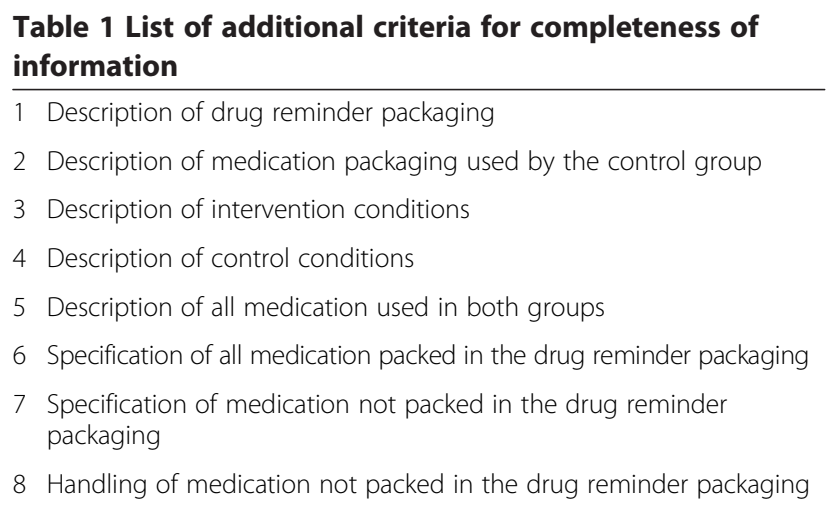

Each criterion was accredited with 1 point; completeness of information was calculated as the sum of the points divided by the number of applicable criteria. clinical outcomes, unless they were part of a composite adherence outcome. A listing of costs was considered as an economic intermediary outcome if compared between groups. Patient surveys on handling, opinion or satisfaction with drug reminder packaging were considered as humanistic intermediary outcomes if comparison between groups was given.

\section{Results}

Of the total 855 identified references, 30 fulfilled the inclusion criteria. The PRISMA flow diagram of study inclusion and the PRISMA checklist are provided in Additional files 1 and 2, respectively. According to the EPHPP assessment tool for study design, 10 studies were RCTs, 19 controlled clinical trials and 1 was a cohort study (one group with a pre- and post-intervention comparison). Compared to the previously published reviews [18-20], a total of 13 studies were additionally included, from which 7 were controlled clinical trials, 5 RCTs and 1 was a cohort study.

Overall, the mean number of participants was 191 (range 14 to 2,081 participants). They were on average 62 years old (range 38 to 87 years, not described (n.d.) in five studies), took an average of 3.9 medications (range 1 to 9 medications, n.d. in 12 studies) and were treated for hypertension (7), diabetes mellitus type 2 (3), geriatric conditions (3), Helicobacter pylori infection (2), HIV (2), vitamin supplementation (2), chronic mental illness (2), hypercholesterolemia (1), epilepsy (1), pain relief in cancer patients (1), anticoagulation (1), and Chlamydia infection (1). Medical conditions were not described in six studies of mainly elderly multimorbid patients. The mean study duration was 5.4 months (range 7 days to 14 months, n.d. in three studies). Table 2 is a summary of the studies.

\section{Effect on adherence}

Considerable variation exists between studies regarding definitions, measures and calculations of adherence. Taking adherence was estimated in 27 studies (90\%). Pill count (15 studies) and patient self-report (12 of which 1 was electronic) were the most used measures. Other methods included refill data (6), therapeutic drug monitoring (5), appointment keeping (2) and clinical measures (2). Eleven studies used composite adherence measures. The calculation of adherence was unclear in three studies [42,47,57].

A significant effect of drug reminder packaging was reported in 17 studies and concerned at least one of the measured adherence parameters. Six of these 17 studies were not incorporated in the previous reviews (Table 2).

Twelve studies reported significant adherence improvement in the group with drug reminder packaging as part of a multiple intervention strategy [30,31,37,40,41,49,50,53-57]. 
Table 2 Summary of the $\mathbf{3 0}$ studies included

\begin{tabular}{|c|c|c|c|c|c|c|c|c|c|c|}
\hline Number & $\begin{array}{l}\text { Lead } \\
\text { author }\end{array}$ & Design & $n$ & Duration & Intervention & $\begin{array}{l}\text { Drug reminder } \\
\text { packaging }\end{array}$ & Outcomes & Effect & $\begin{array}{l}\text { Methodological } \\
\text { quality }\end{array}$ & $\begin{array}{l}\text { Completeness } \\
\text { of information }\end{array}$ \\
\hline 1 & Ascione [30] & $\mathrm{CCT}$ & 158 & n.d. & $\begin{array}{l}\text { Drug reminder packaging, } \\
\text { counseling }\end{array}$ & n.d. & A: Self-report*: & Unclear & Strong & 0.13 \\
\hline \multirow[t]{4}{*}{2} & Azrin [31] & $\mathrm{CCT}$ & 39 & $2 \mathrm{~m}$ & $\begin{array}{l}\text { a. Drug reminder packaging, } \\
\text { counseling with family member } \\
\text { vs }\end{array}$ & $\begin{array}{l}\text { Multicompartment } \\
\text { adherence aid }\end{array}$ & A: Pill count*: & $\begin{array}{l}\text { a. vs baseline: } 95.03 \text { vs } \\
76.24(P<0.05, \varnothing \mathrm{Cl})\end{array}$ & Strong & 0.13 \\
\hline & & & & & $\begin{array}{l}\text { b. Drug reminder packaging, } \\
\text { counseling vs }\end{array}$ & & & $\begin{array}{l}\text { b. vs baseline: } 92.01 \text { vs } \\
69.52(P<0.01, \varnothing \text { CI })\end{array}$ & & \\
\hline & & & & & c. Psychoeducational condition & & & c. vs baseline: n.s. & & \\
\hline & & & & & & & $\begin{array}{l}\text { C: Symptoms } \\
\text { checklist 90-R: }\end{array}$ & n.s. & & \\
\hline \multirow[t]{2}{*}{3} & Becker [32] & $\mathrm{CCT}$ & 180 & $12 \mathrm{~m}$ & Drug reminder packaging & Multidrug punch card & $\begin{array}{l}\text { A: Pill count, self- } \\
\text { report, BP: }\end{array}$ & n.s. & Moderate & 0.38 \\
\hline & & & & & & & C: BP: & n.s. & & \\
\hline \multirow[t]{4}{*}{4} & Binstock [33] & $\mathrm{CCT}$ & 112 & $12 \mathrm{~m}$ & a. Counseling vs & n.d. & A: Self-report: & a., b., c.: n.s. & Weak & 0.25 \\
\hline & & & & & $\begin{array}{l}\text { b. Drug reminder packaging, } \\
\text { counseling vs }\end{array}$ & & $C: \mathrm{sBP}^{*}, \mathrm{dBP} P^{*}$ & $\begin{array}{l}\text { b. vs a.: } 133 / 80 \mathrm{mmHg} \text { vs } \\
148 / 89 \mathrm{mmHg}(P<0.01 \\
\varnothing \mathrm{Cl})\end{array}$ & & \\
\hline & & & & & $\begin{array}{l}\text { c. Drug reminder packaging, } \\
\text { counseling, other aids vs other } \\
\text { interventions }\end{array}$ & & & $\begin{array}{l}\text { C. vs a.: } 134 / 84 \mathrm{mmHg} \text { vs } \\
148 / 89 \mathrm{~mm} \mathrm{Hg}(P<0.01 \text {, } \\
\varnothing \text { Cl) }\end{array}$ & & \\
\hline & & & & & & & & b. vs c.: n.s. & & \\
\hline 5 & $\begin{array}{l}\text { Crome } \\
\text { (1980) [34] }\end{array}$ & $\mathrm{CCT}$ & 26 & $10 \mathrm{~d}$ & Drug reminder packaging & $\begin{array}{l}\text { Multicompartment } \\
\text { adherence aid }\end{array}$ & A: Pill count: & n.s. & Weak & 0.25 \\
\hline 6 & $\begin{array}{l}\text { Crome } \\
\text { (1982) [35] }\end{array}$ & $\mathrm{CCT}$ & 78 & $4 w$ & Drug reminder packaging & Multidrug punch card & A: Pill count: & n.s. & Weak & 0.25 \\
\hline \multirow[t]{3}{*}{7} & $\begin{array}{l}\text { Eshelman } \\
{[36]}\end{array}$ & $\mathrm{CCT}$ & 100 & n.d. & Drug reminder packaging & n.d. & A: TDM*: & $\begin{array}{l}\text { 'Adherent' patients: } 97 \% \\
\text { vs } 69 \%(P<0.05, \varnothing \mathrm{Cl})\end{array}$ & Moderate & 0.13 \\
\hline & & & & & & & Pill count: & n.s. & & \\
\hline & & & & & & & Self-report: & Unclear & & \\
\hline \multirow[t]{3}{*}{8} & Fairley [37] & $\mathrm{RCT}$ & 43 & $5 \mathrm{~m}$ & $\begin{array}{l}\text { Drug reminder packaging, } \\
\text { counseling, other aids }\end{array}$ & $\begin{array}{l}\text { Multicompartment } \\
\text { adherence aid }\end{array}$ & A: Self-report*: & $\begin{array}{l}\text { Total Morisky score: } 3.3 \text { vs } \\
2.9(P=0.006, \varnothing \mathrm{Cl})\end{array}$ & Moderate & 0.13 \\
\hline & & & & & & & & $\begin{array}{l}\text { Rate of patients with a } \\
\text { Morisky score of 0: } 29 \% \text { vs } \\
49 \%(P=0.04, \varnothing \text { Cl) }\end{array}$ & & \\
\hline & & & & & & & $\begin{array}{l}\text { C: CD4 cell count, } \\
\text { viral load: }\end{array}$ & n.s. & & \\
\hline
\end{tabular}


Table $\mathbf{2}$ Summary of the $\mathbf{3 0}$ studies included (Continued)

Multidrug punch card A: Pill count + self-

n.s.

report:

$\mathrm{C:}{ }^{13} \mathrm{C}-\mathrm{UBT}$ :

n.s.

$\begin{array}{llll}\begin{array}{l}\text { Huang } \\ \text { (TRACE) [39] }\end{array} & \text { RCT } & 184 & 2 \mathrm{~m} \\ \begin{array}{l}\text { Huang } \\ \text { (VITAL) [39] }\end{array} & \text { CCT } & 297 & \text { Unclear }\end{array}$

Drug reminder packaging

Drug reminder packaging

(multidrug punch card vs multicompartment adherence aid)

Multicompartment
adherence aid
Multidrug punch
card,
multicompartment
adherence aid

A: Pill count, self-

report, TDM:

n.s.

Moderate

A: Pill count*:

Patients who took $>90 \% \quad$ Moderate of pills: $93 \%$ vs $87 \%$ $(P=0.05, \varnothing \mathrm{Cl})$

Positive answer to

question 'forgot to take pills': $21 \%$ vs $31 \%$

$(P=0.05, \varnothing \mathrm{Cl})$; self-report total score: n.s.

TDM

n.s.

Drug reminder packaging counseling, regular follow-up

Multidrug punch card A: Pill count*

C: $\mathrm{SBP}^{*}$ :

$\mathrm{dBP} *$

LDL-C*:

Drug reminder packaging counseling, other aids
95.5 vs $69.1(P<0.001$

Strong $\varnothing \mathrm{Cl})$

Drug reminder packaging vs baseline: $-6.9 \mathrm{mmHg}$ $(P=0.005, C l-10.7-(-3.1)$ $\mathrm{mm} \mathrm{Hg}$ )

Drug reminder packaging vs baseline: $-2.5 \mathrm{~mm} \mathrm{Hg}$ $P=0.04, \mathrm{Cl}-4.9-(-0.2)$ $\mathrm{mm} \mathrm{Hg}$

Drug reminder packaging vs baseline at $8 \mathrm{~m}$ :

$-4.8 \mathrm{mg} / \mathrm{dl}(P=0.001$ $\mathrm{Cl}-7.8-(-1.9) \mathrm{mg} / \mathrm{l})$

Drug reminder packaging vs baseline at $14 \mathrm{~m}$ : n.s.

TT1 (patients unavailable Weak

for follow-up took 100\%

[cg] or $0 \%$ [ig] of drugs):

No. of patients with $>60 \%$ of pills taken: n.s.

Patients with $>90 \%$ of

pills taken: $87 \%$ vs $71 \%$

$(P<0.05, \varnothing \mathrm{Cl})$

IT2 (patients unavailable

for follow-up took $0 \%$

$[\mathrm{cg}+\mathrm{ig}]$ of drugs): 


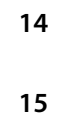

\section{Multicompartment adherence aid \\ Multicompartmen \\ Multicompartment adherence aid}

Multicompartment adherence aid

Appropriate
prescriptions*:

Change in total

amount of opioids prescribed and taken:

Patients with $>60 \%$ of

pills taken: $94 \%$ vs $78 \%$

$(P<0.05, \varnothing \mathrm{Cl})$

Patients with $>90 \%$ of

pills taken: $87 \%$ vs $59 \%$

$(P<0.01, \varnothing \mathrm{Cl})$

C: (Proxies for

adherence)

hospitalizations*:

infections*:

C: Pain reduction*:

Weak

$-0.74 \%$ vs $-0.53 \%$

$(P<0.0001, \varnothing(1)$

$75.8 \%$ vs $39.3 \%(\varnothing P, C l)$

Weak

Drug reminder packaging

s baseline: $75.8 \%$ vs

46.8\% $(P<0.01, \varnothing \mathrm{Cl})$

Drug reminder packaging

vs baseline: $76.1 \%$ vs

56.7\% $(P<0.05, \varnothing \mathrm{Cl})$

0.33 vs $1.04(P<0.05, \varnothing$

(I)

Reduction with increased

medication intake

$(\varnothing$ numbers given,

$$
P<0.05, \varnothing(\mathrm{Cl})
$$

Relieve in average, worst Moderate and least pain: $\varnothing$ numbers

given $(P<0.0001, \varnothing \mathrm{Cl})$

Patients with appropriate

opioid analgesic

prescriptions vs baseline:

$37.0 \%$ vs $28.3 \%(P=0.008$

$\varnothing \mathrm{Cl})$

Prescribed: $\varnothing$ numbers given $(P<0.0001, \varnothing \mathrm{Cl})$

Taken: $\varnothing$ numbers given $(P<0.001, \varnothing \mathrm{Cl})$

92.6 vs $79(P<0.0001$

Weak 
Nochowitz Pre- $\quad 14 \quad 3$ m

[47] post-

post-

20

Park [48] CCT $61 \quad 2 \mathrm{w}$

Peterson [49] $\mathrm{RC}$

$4 \mathrm{~m}$

Rheder [50] CCT $100 \quad 3 \mathrm{~m}$

Schneider

[51]
Drug reminder packaging \pm organizing chart, factorial

Drug reminder packaging, counseling, other aids

Drug reminder packaging \pm counseling, factorial

Drug reminder packaging, other aids

Drug reminder packaging \pm
counseling, factorial
Multicompartment
adherence aid

Multicompartment adherence aid

Multicompartment

adherence aid

Multicompartment A: MRF

keeping:

$\mathrm{C}: \mathrm{BP} *$
Multidrug punch card A: MPR *

No. of patients reporting

all medication taken: 9 vs

$8(\varnothing P, C l)$

A: Pill count $(+/-$ self- n.s.

Moderate

report if pills were

not available):

Sub-therapeutic INR

values $(<2)$ vs baseline:

$35 \%$ vs $60 \%(P=0.04$

$\varnothing \mathrm{Cl})$

Time spent in therapeutic

range vs baseline: $56 \%$ v

$32 \%(P=0.03, \varnothing \mathrm{Cl})$

A: Electronic self-

Unclear

Weak

'Adherent' patients: 88\%

vs $50 \%(P<0.01, \varnothing \mathrm{Cl})$

Moderate

Patients within

therapeutic range vs

baseline: $88 \%$ vs $48 \%$

$(P<0.005, \varnothing \mathrm{Cl})$

Appointment n.s.

C: Seizure frequency*: Frequency of seizures vs baseline: 2.5 vs $6(P<0.01$

$\varnothing \mathrm{Cl})$

A: Pill count*:

No. of patients who took Weak

$\geq 95 \%$ of pills: Drug

reminder packaging $\pm \mathrm{mi}$

$>\mathrm{mi}, \varnothing$ numbers given

$(P<0.01, \varnothing \mathrm{Cl})$

Drug reminder packaging + mi vs baseline: $\varnothing$ numbers given $(P<0.02$,

$\varnothing \mathrm{Cl})$

Drug reminder packaging vs baseline: n.s.

0.93 vs $0.87(P=0.039, \quad$ Moderate $\varnothing \mathrm{Cl})$

Patients with their

prescription refilled

on-time ( $\pm 5 \mathrm{~d}): 80.4 \%$ vs

$66.1 \%(P=0.012, \varnothing \mathrm{Cl})$ 
Drug reminder packaging \pm videotape \pm telephone interview, factorial

Drug reminder packaging counseling, other aids

\section{(per capita)}

Drug reminder packaging $\pm \mathrm{rr}$, factorial
Unit-of-use packaging $\quad$ A: MPR*

Prescription expenditure*.

All other expenditure:

$\begin{array}{ll} & \begin{array}{l}r r+\text { drug reminder } \\ \text { packaging vs cg: }\end{array} \\ \text { Prescription } & +\$ 104.39(P \leq 0.05, \varnothing \mathrm{Cl}) \\ \text { expenditure*: } & \\ \text { Physician } & -\$ 78.41(P \leq 0.05, \varnothing \mathrm{Cl}) \\ \text { expenditure*: } & \end{array}$

Hospital expenditure*: $-\$ 89.54(P \leq 0.05, \varnothing \mathrm{Cl})$

Laboratory

expenditure:

Total expenditure*: $\quad-\$ 75.28(P \leq 0.05, \varnothing \mathrm{Cl})$

\section{(per capita)}

Unit-of-use packaging A: Self-report (noncompliance score) ${ }^{*}$ packaging vs drug
Drug reminder packaging Weak vs cg: 0.67 vs 0.56

$\mathrm{rr}+$ drug reminder packaging vs cg: 0.79 vs $0.56(P \leq 0.05, \varnothing \mathrm{C})$

ir + drug reminder vs 0.67 (P packaging: 0.79 $0.67(P \leq 0.05, \varnothing \mathrm{Cl})$

Drug reminder packaging vs cg:

$\$ 48.17(P \leq 0.05, \varnothing C \mid)$ n.s.

Drug reminder packaging Moderate vs cg: 30.2 vs 50.7

$(P<0.001, \varnothing \mathrm{Cl})$

Drug reminder packaging

+ videotape vs cg: 5.5 vs

$11.1(P<0.001, \varnothing \mathrm{Cl})$

At $6 \mathrm{~m}: 0.91$ vs 0.64

Moderate

< $0.0001, \varnothing \mathrm{Cl})$

At $12 \mathrm{m:} 0.86$ vs 0.62

$(P<0.0001, \varnothing \mathrm{Cl})$
0.33

\begin{tabular}{|c|c|c|c|c|c|c|}
\hline \multirow[t]{2}{*}{$\begin{array}{l}\text { Valenstein } \\
{[56]}\end{array}$} & \multirow[t]{2}{*}{$\mathrm{RCT}$} & \multirow[t]{2}{*}{118} & $12 \mathrm{~m}$ & \multirow[t]{2}{*}{$\begin{array}{l}\text { Drug reminder packaging, } \\
\text { counseling, other aids }\end{array}$} & \multirow[t]{2}{*}{ Multidrug punch card A: MPR* } & $\begin{array}{l}\text { At } 6 \mathrm{m:} 0.91 \text { vs } 0.64 \\
(P<0.0001, \varnothing \mathrm{Cl})\end{array}$ \\
\hline & & & & & & $\begin{array}{l}\text { At } 12 \mathrm{~m}: 0.86 \text { vs } 0.62 \\
(P<0.0001, \varnothing \mathrm{Cl})\end{array}$ \\
\hline
\end{tabular}


Table 2 Summary of the 30 studies included (Continued)

\begin{tabular}{|c|c|c|c|c|c|c|c|c|c|c|}
\hline & & & & & & & $\mathrm{CAM}^{*}:$ & \multirow{2}{*}{$\begin{array}{l}\text { At } 6 \mathrm{~m}: 26 \% \text { vs } 9 \% \\
(P=0.0003, \varnothing \mathrm{Cl})\end{array}$} & & \\
\hline & & & & & & & $\begin{array}{l}\text { (MPR + self-report + } \\
\text { TDM) }\end{array}$ & & & \\
\hline & & & & & & & & $\begin{array}{l}\text { At } 12 \mathrm{m:}: 17 \% \text { vs } 9 \% \\
(P=0.06, \varnothing \mathrm{Cl})\end{array}$ & & \\
\hline & & & & & & & $\begin{array}{l}\text { C: Psychiatric } \\
\text { symptoms: }\end{array}$ & n.s. & & \\
\hline & & & & & & & $\begin{array}{l}\text { H: Patient satisfaction, } \\
\text { quality of life: }\end{array}$ & n.s. & & \\
\hline \multirow[t]{6}{*}{29} & \multirow[t]{6}{*}{ Ware [57] } & \multirow[t]{6}{*}{$\mathrm{CCT}$} & \multirow[t]{6}{*}{84} & \multirow[t]{6}{*}{$3 \mathrm{~m}$} & \multirow[t]{6}{*}{$\begin{array}{l}\text { Drug reminder packaging, } \\
\text { counseling }\end{array}$} & \multirow[t]{6}{*}{ Multidrug punch card } & \multirow[t]{6}{*}{$\begin{array}{l}\text { A: Self-report + pill } \\
\text { count: }\end{array}$} & $\begin{array}{l}\text { Patients taking all } \\
\text { prescribed doses: }\end{array}$ & \multirow[t]{6}{*}{ Weak } & \multirow[t]{6}{*}{0.38} \\
\hline & & & & & & & & $\begin{array}{l}\text { At discharge: } 86.7 \% \text { vs } \\
66.7 \%(P=0.03, \varnothing \mathrm{Cl})\end{array}$ & & \\
\hline & & & & & & & & $\begin{array}{l}\text { At } 10 \mathrm{~d}: 69 \% \text { vs } 41 \% \\
(P=0.02, \varnothing \mathrm{Cl})\end{array}$ & & \\
\hline & & & & & & & & $\begin{array}{l}\text { At } 1 \mathrm{m:}: 64.4 \% \text { vs } 38.5 \% \\
(P=0.03, \varnothing \mathrm{Cl})\end{array}$ & & \\
\hline & & & & & & & & $\begin{array}{l}\text { At } 2 \mathrm{m:}: 57.8 \% \text { vs } 28.2 \% \\
(P=0.01, \varnothing \mathrm{Cl})\end{array}$ & & \\
\hline & & & & & & & & $\begin{array}{l}\text { At } 3 \mathrm{~m}: 48.9 \% \text { vs } 23.1 \% \\
(P=0.03, \varnothing \mathrm{Cl})\end{array}$ & & \\
\hline \multirow[t]{6}{*}{30} & \multirow{6}{*}{$\begin{array}{l}\text { Winland- } \\
\text { Brown [58] }\end{array}$} & \multirow[t]{6}{*}{$\mathrm{CCT}$} & \multirow[t]{6}{*}{61} & \multirow[t]{6}{*}{$6 \mathrm{~m}$} & \multirow[t]{6}{*}{ Drug reminder packaging } & \multirow{6}{*}{$\begin{array}{l}\text { Multicompartment } \\
\text { adherence aid }\end{array}$} & A: Pill count: & n.s. & \multirow[t]{6}{*}{ Weak } & \multirow[t]{6}{*}{0.13} \\
\hline & & & & & & & $\begin{array}{l}\text { C: BP, INR, TDM, } \\
\text { mood stabilization, } \\
\text { HbA }_{1 C} \text { : }\end{array}$ & Not reported & & \\
\hline & & & & & & & Physician visits: & $\begin{array}{l}\text { Mean (per patient) vs } \\
\text { baseline: } 1.5 \text { vs } 1.5 \\
(\varnothing P, C I)\end{array}$ & & \\
\hline & & & & & & & Hospital admission: & $\begin{array}{l}\text { No. of patients vs } \\
\text { baseline: } 7 \text { vs } 4 \text { ( } \varnothing \text { P, Cl) }\end{array}$ & & \\
\hline & & & & & & & Home visit: & $\begin{array}{l}\text { No. of patients vs } \\
\text { baseline: } 0 \text { vs } 0 \text { ( } \varnothing P, C l)\end{array}$ & & \\
\hline & & & & & & & $\begin{array}{l}\text { Transition to a higher } \\
\text { level of care: }\end{array}$ & Not reported & & \\
\hline
\end{tabular}

The 13 additional studies compared to previous reviews [18-20] are designated in bold. * indicates statistically significant outcomes.

${ }^{13} \mathrm{C}-\mathrm{UBT},{ }^{13} \mathrm{C}$-urea breath test; $\mathrm{A}$, adherence outcome; (s, d)BP, (systolic, diastolic) blood pressure; $\mathrm{C}$, clinical outcome; $\mathrm{CAM}$, composite adherence measure; $\mathrm{CCT}$, controlled clinical trial; $\mathrm{cg}$, control group; $\mathrm{Cl}$, confidence interval; d, days; $\mathrm{E}$, economic outcomes; $\mathrm{H}$, humanistic outcomes; $\mathrm{HbA}_{1 \mathrm{C}}$, glycated hemoglobin; ig, intervention group; INR, international normalized ratio; LDL-C, low-density lipoprotein

cholesterol; $\mathrm{m}$, months; mi, multiple interventions; MRC, medication refill compliance; MRF, medication refill frequency; No., number; $\mathrm{n} . \mathrm{s}$, not significant; $\mathrm{RCT}$, randomized controlled trial; rr, refill reminder; TDM, therapeutic drug monitoring; $w$, weeks. 
The effect on adherence was also significant when drug reminder packaging was a single intervention [36,39,44,46,50,51,53-55]; however, it was less pronounced in direct comparison with multiple interventions [50,53-55].

Methodological quality and completeness of information Methodological quality was rated as strong for 5 studies, moderate for 12 and weak for 13. Overall, weaknesses were in the methods used for data collection (mostly not valid and not reliable) and the report of confounders and their comparison between groups (insufficient or missing). The most accurate standard in statistical analysis, the intention-to-treat analysis, was applied by seven studies. The number of studies with strong and moderate methodological quality doubled after 1996, the year of the first publication of the CONSORT statements [59], while the number of weak methodological quality studies diminished by a factor of 3 .

Completeness of information ranged from 0 to 1.0 with a mean score of 0.3 . Two studies [40,55] gave complete information for all required details. Reported criteria for the completeness of information are depicted in Figure 1. Criteria 7 and 8 were not applicable for 5 studies [40,53-56] and practically non-existent in all 25 remaining studies (criterion 7: 0; criterion 8: 1). Information on the person in charge and place of intervention were often missing from the description of the intervention and control conditions. Figure 2 shows the included studies according to their methodological quality, completeness of information and outcome measures.

\section{Outcomes}

Two studies assessed direct costs as intermediary economic outcomes [53,54] and there was a significant increase in prescription costs. However, a cost-effectiveness analysis that would qualify as an economic outcome according to the ECHO model was not reported.

Clinical outcomes were measured in 16 studies using one or several parameters: blood pressure (6), glycated hemoglobin $\left(\mathrm{HbA}_{1 \mathrm{C}}\right)(2)$, psychiatric symptoms (2), lowdensity lipoprotein cholesterol (LDL-C) levels (1), pain reduction (1), number of seizures (1), plasma levels of anticonvulsant drugs (1), viral load (1), CD4 cell count (1), number of opportunistic infections (1), hospitalizations (1), percentages of sub-therapeutic international normalized ratio (INR) values (1), time within the therapeutic INR range (1) and ${ }^{13} \mathrm{C}$-urea breath test (1).

Of these 16 studies, 7 were not incorporated in the previous reviews. Five of the seven additional studies showed a statistically significant effect [40,43-45,47]. In one study, LDL-C levels and blood pressure were significantly reduced after eight months compared to the baseline for patients using drug reminder packaging (LDL-C: $-4.8 \mathrm{mg} / \mathrm{dl}$, $P=0.001$; systolic blood pressure: $-6.9 \mathrm{mmHg}, P=0.005$; diastolic blood pressure: $-2.5 \mathrm{mmHg}, P=0.04$ ) [40]. In a study with diabetes mellitus type 2 patients, $\mathrm{HbA}_{1 \mathrm{C}}$ was significantly reduced $(-0.74 \%, P<0.0001)$ and patients who took $\geq 5$ tablets/day, $\geq 3$ hypoglycemic drugs/day and were $<55$ years old had the largest benefit from drug reminder packaging [43]. In other studies, pain reduction was effective in cancer patients $(P<0.0001)$ [45], the number of opportunistic infections and hospitalizations decreased significantly in HIV patients $(P<0.05)$ [44], the percentages of sub-therapeutic INR values with oral anticoagulation (warfarin) decreased $(P=0.04)$ and time within the therapeutic INR range increased significantly $(P=0.03)$ [47]. Of the ten studies with multiple adherence-enhancing strategies in the intervention group, six showed significantly improved clinical outcomes $[33,40,45,47,49,50]$. The clinical outcomes of all studies are presented in Table 2.

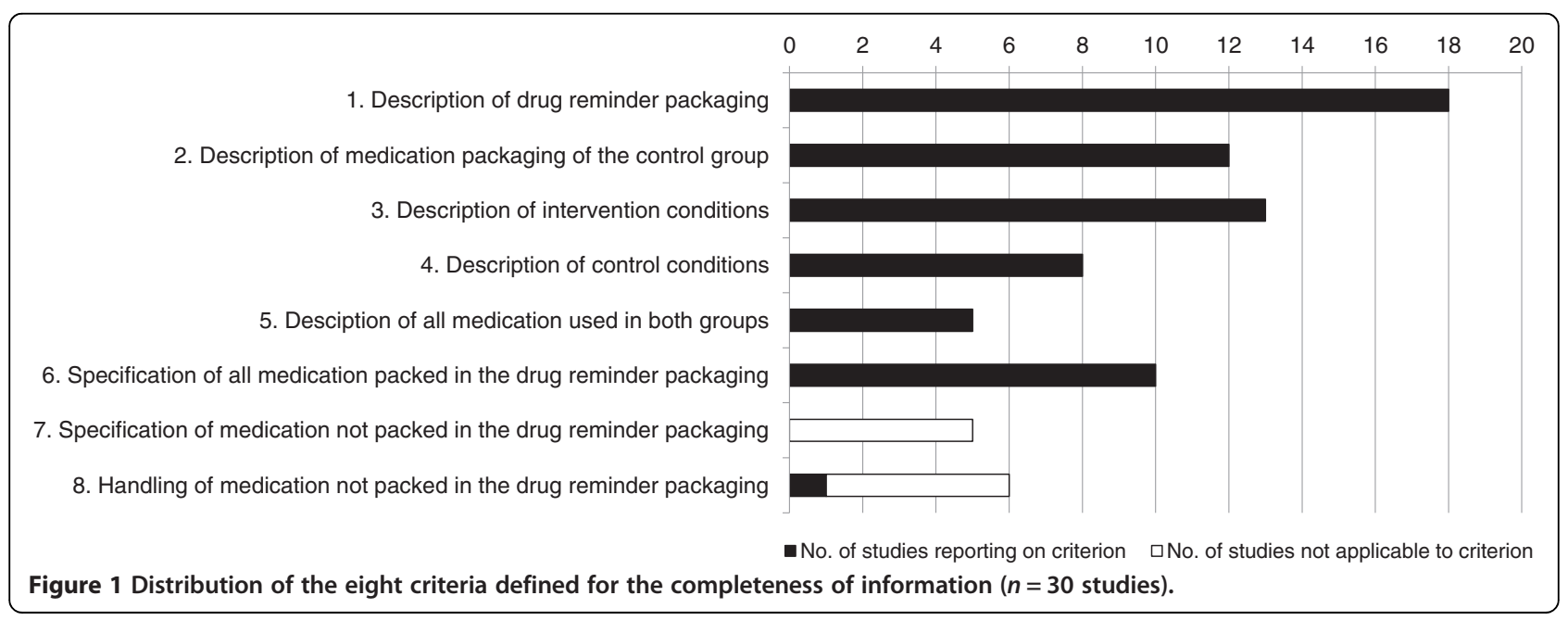




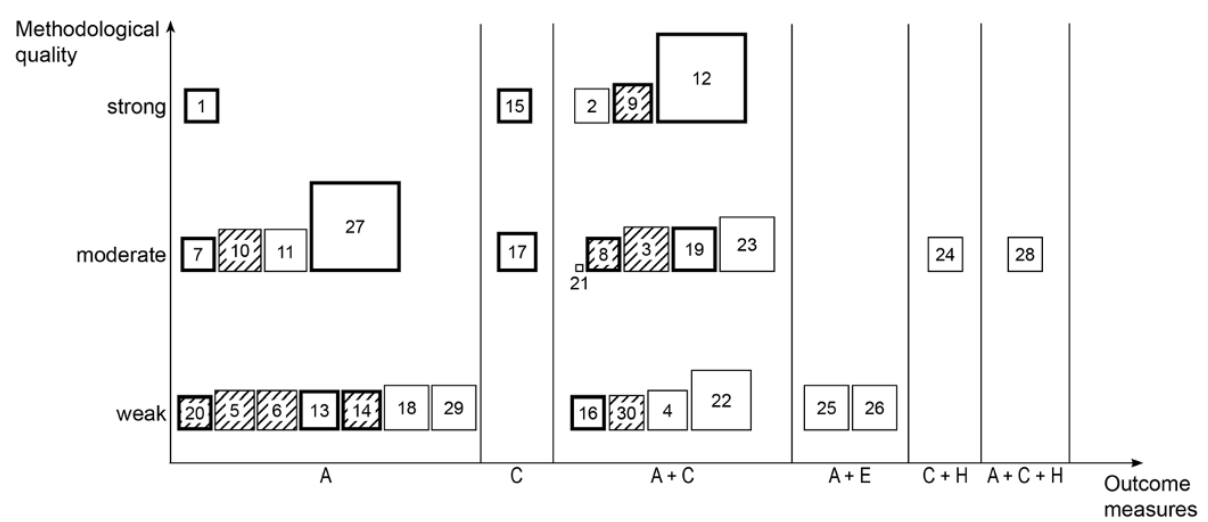

Figure 2 Consolidation of results of outcomes, methodological quality and completeness of information. Each box represents one study numbered as in Table 2, plotted in a segment of reported outcome(s) and at a height based on its methodological quality. Completeness of information is indicated by the size of the box, with values between 0 (e.g., study no. 21) and 1 (e.g., study no. 12). Bold frames are for the additionally included studies compared to previously published reviews [18-20]. No filling indicates at least one outcome was statistically significant and shading indicates none of the outcomes were statistically significant. A, adherence; $\mathrm{C}$, clinical outcome; $\mathrm{E}$, economic outcome; $\mathrm{H}$, humanistic outcome.

Two studies reported humanistic outcomes [52,56]. The usability of drug reminder packaging was rated significantly higher than the usability of usual packaging [52]. Safety issues related to the intervention were addressed by two studies $[49,56]$.

Clear gaps emerged from the overall results. Aside from methodological weaknesses (under-reporting of quality issues) and incomplete information (under-reporting of control settings and specification of medication), economic outcomes (cost-effectiveness), humanistic outcomes and safety issues are lacking.

\section{Discussion}

Although more than half of the studies included in this review reported significant effects, only three studies were graded as methodologically strong. Drug reminder packaging had a significant effect on adherence in a geriatric population [30], for chronic mental illness [31] and for cardiovascular disease [40]. The overall effect of drug reminder packaging on adherence parameters remains inconclusive, as reported by previous reviews with more restrictive selection criteria [18-20]. Three studies reported a significant effect on adherence but not on clinical outcomes $[31,50,56]$. Thus, the question of how much adherence is necessary for altering treatment success is raised and there is a requirement to present the clinical benefits for the patients [60]. We observed that drug reminder packaging offers a broad field of application and is mostly used for polypharmacy. As a consequence, disease-unspecific, generalizable clinical outcomes like morbidity or re-hospitalization rates would provide viable and comparable results rather than measures of diseasespecific clinical parameters. Only two trials investigated such outcomes [44,58], with one showing that drug reminder packaging significantly reduced the mean hospitalization rate.

We included five RCTs in the evidence map that were excluded by three previous reviews [18-20] because of their multiple intervention design. In a direct comparison (factorial trials), the effect was higher with multiple interventions, which is consistent with previous findings $[14,18]$. Yet, the evidence is limited, for these trials were graded as weak in methodological quality.

The overall methodological quality of the studies included is poor and thus evidence for the effect of drug reminder packaging on adherence is low. We used a quality assessment tool that is applicable to a variety of study designs and was specifically developed to provide research evidence for studies on public health services with a focus on behavior change education [24]. In comparison to previous reviews, we were able to include four additional studies of strong methodological quality $[30,38,40,43]$. However, information on intervention and control settings was incomplete in three of these additional studies (completeness scores: $0.13, n=2 ; 0.25$, $n=1)$. As a consequence, being graded as strong and complying with all the criteria for completeness of information was observed in one out of the 30 studies included [40]. It therefore represents a thin basis for informed clinical decision support.

The increasing number of methodologically strong trials after 1996, the year when the CONSORT statements were released, is intriguing and probably follows from under-reporting in studies published before 1996. Various authors indeed stated that complete reporting of methodological quality according to the CONSORT criteria was inadequate, but that poor reporting did not necessarily correlate with the quality of how the trial was conducted [61-64]. The CONSORT statements of 
non-pharmacological treatment require 'precise details of both, the experimental treatment and the comparator' [27] and omission of trial details has been shown to lead to decreased uptake of trial results into clinical practice $[65,66]$. Thus, to obtain valuable and reliable study results, high methodological quality and detailed information are crucial.

Most studies were designed as RCTs, which provide the most reliable results through the minimization of confounding. However, RCTs might not be the appropriate design for all research questions and settings, especially in the field of behavior research. Alternative designs might be worth considering. Firstly, randomized allocation of study participants to a predefined intervention may not be practicable since tailored interventions, in respect of patients' needs and abilities, are expected to be the most effective [14]. Secondly, in studies on survival outcomes for HIV patients, investigating adherence-enhancing strategies in a randomized controlled fashion has been declared to be ethically difficult $[67,68]$. The reason for this declaration was the assumption that allocation to the control condition equaled withholding a tool, which could possibly lead to higher survival rates through an optimal clinical response due to increased adherence $[67,68]$. Thirdly, behavioral interventions are often complex and can only be controlled poorly under real-life conditions and therefore randomization might not be practical in a primary care setting [69]. Consequently, confounding could even persist despite randomization. Alternatives to conventional randomization designs, i.e., randomization at the patient level, include pre- and postcohort studies, historical control studies, pre-randomized designs and cluster randomization [70].

More studies could be included and research gaps identified using our approach of evidence mapping. Patientrelevant disease-unspecific long-term clinical outcomes, e.g., (re-)hospitalization, admission to a nursing home, etc., were neglected. Economic outcomes as defined by Kozma et al. [29] were not reported in any study on drug reminder packaging. This may be due to the fact that drug reminder packaging is generally supposed to be inexpensive, and thus cost-effective. Humanistic outcomes were measured in two studies $[52,56]$, which is insufficient for judging whether a condition optimally treated through drug reminder packaging leads to increased quality of life. Improved adherence could lead to increased adverse events as well. However, safety issues were reported by two studies only $[49,56]$. Patient satisfaction and other aspects of safety, such as opening medication packaging, confusion with new packaging and decreased ability to identify one's own medication [71-74], were hardly mentioned by the studies.

Our study has strengths. First, evidence mapping allows the inclusion of more studies and gives an overall view of the subject. Second, the tool used to assess methodological quality is independent of study design (EPHPP) and was developed specifically to assess studies within the scope of public health. Third, with completeness of information, a further element for judging quality is added. Fourth, the consolidation of adherence outcomes and economic, clinical and humanistic parameters allows an overall presentation and highlights research gaps. Our study has limitations also, such as the language restriction, which led to the exclusion of articles considered relevant. Information may also have been missed due to the exclusion of studies performed in developing countries.

A suggestion for future research is to develop methodologically strong studies reporting complete information to clarify the effect of drug reminder packaging on medication adherence.

\section{Conclusions}

New information was extracted from the 30 studies included and several studies had statistically significant and relevant results for adherence and clinical outcomes with drug reminder packaging. However, firm conclusions cannot be given for the effect of drug reminder packaging on adherence, mainly because the studies lack methodological quality and the information was incomplete. The main research gaps concerned economic, disease-unspecific clinical outcomes and humanistic outcomes. Safety issues and satisfaction with the intervention were marginally reported. Researchers of behavioral interventions might consider alternative study designs for similar research questions, without neglecting methodological issues and reporting important details. Future research should aim at filling the observed gaps with a focus on patient safety and the benefit to patients as well as on implementable and valuable interventions. Drug reminder packaging should be distributed with respect to patient needs, requests and abilities.

\section{Additional files}

\section{Additional file 1: PRISMA flow diagram.}

Additional file 2: PRISMA check list.

\begin{abstract}
Abbreviations
Cl: confidence interval; CONSORT: Consolidated Standards of Reporting Trials; ECHO: Economic, Clinical and Humanistic Outcomes; EPHPP: effective public health practice project; $\mathrm{HbA}_{1}$ : glycated hemoglobin; HIV: human immunodeficiency virus; INR: international normalized ratio; LDL-C: low-density lipoprotein cholesterol; MeSH: medical subject headings; n.d.: not described; PRISMA: Preferred Reporting Items for Systematic Reviews and Meta-analyses; $\mathrm{RCT}$ : randomized controlled trial.
\end{abstract}

Competing interests

The authors declare that they have no competing interests. 


\section{Authors' contributions}

FB designed the review protocol, carried out the literature search, extracted data from selected studies, conducted quality assessments and drafted the manuscript. ES reviewed the literature search and the quality assessment. KS participated in the conception of the review and revised the manuscript critically for intellectual content. KEH participated in the design of the review, helped to draft the manuscript and revised it critically for intellectual content. IA participated in the design of the review, helped to draft the manuscript and revised it critically for intellectual content. All authors read and approved the final manuscript.

\section{Acknowledgements}

The authors thank Emily Schultz for helpful comments in the preparation of this manuscript. This work was funded by the Pharmaceutical Care Research Group, Basel, Switzerland.

\section{Author details}

'Pharmaceutical Care Research Group, Department of Pharmaceutical Sciences, University of Basel, Klingelbergstrasse 50, CH-4056 Basel, Switzerland. ${ }^{2}$ University Hospital Basel, Hospital-Pharmacy, Spitalstrasse 26, $\mathrm{CH}-4031$ Basel, Switzerland.

Received: 10 December 2013 Accepted: 13 March 2014 Published: 24 March 2014

\section{References}

1. Adherence to long-term therapies - Evidence for action. [http://www. who.int/chp/knowledge/publications/adherence_full_report.pdf]

2. DiMatteo MR: Variations in patients' adherence to medical recommendations: a quantitative review of 50 years of research. Med Care 2004, 42:200-209.

3. Cramer JA, Rosenheck R: Compliance with medication regimens for mental and physical disorders. Psychiatr Serv 1998, 49:196-201.

4. Osterberg L, Blaschke T: Adherence to medication. N Engl J Med 2005, 353:487-497.

5. Wroe AL: Intentional and unintentional nonadherence: a study of decision making. J Behav Med 2002, 25:355-372.

6. Cutler DM, Long G, Berndt ER, Royer J, Fournier A-A, Sasser A, Cremieux P: The value of antihypertensive drugs: a perspective on medical innovation. Health Aff (Millwood) 2007, 26:97-110.

7. Ho PM, Magid DJ, Shetterly SM, Olson KL, Maddox TM, Peterson PN, Masoudi FA, Rumsfeld JS: Medication nonadherence is associated with a broad range of adverse outcomes in patients with coronary artery disease. Am Heart J 2008, 155:772-779.

8. Ho PM, Rumsfeld JS, Masoudi FA, McClure DL, Plomondon ME, Steiner JF, Magid DJ: Effect of medication nonadherence on hospitalization and mortality among patients with diabetes mellitus. Arch Intern Med 1836-1841, 2006:166.

9. Zed PJ, Abu-Laban RB, Balen RM, Loewen PS, Hohl CM, Brubacher JR, Wilbur K, Wiens MO, Samoy LJ, Lacaria K, Purssell RA: Incidence, severity and preventability of medication-related visits to the emergency department: a prospective study. Can Med Assoc J 2008, 178:1563-1569.

10. Hughes DA, Bagust A, Haycox A, Walley T: The impact of non-compliance on the cost-effectiveness of pharmaceuticals: a review of the literature. Health Econ 2001, 10:601-615.

11. Roebuck MC, Liberman JN, Gemmill-Toyama M, Brennan TA: Medication adherence leads to lower health care use and costs despite increased drug spending. Health Aff (Millwood) 2011, 30:91-99.

12. Leendertse AJ, Egberts AC, Stoker $\sqcup$, van den Bemt PM: Frequency of and risk factors for preventable medication-related hospital admissions in the Netherlands. Arch Intern Med 2008, 168:1890-1896.

13. Mennini FS, Marcellusi A, von der Schulenburg JM, Gray A, Levy P, Sciattella P, Soro M, Staffiero G, Zeidler J, Maggioni A, Schmieder RE: Cost of poor adherence to anti-hypertensive therapy in five European countries. Eur $J$ Health Econ 2014. Epub ahead of print.

14. Haynes RB, Ackloo E, Sahota N, McDonald HP, Yao X: Interventions for enhancing medication adherence. Cochrane Database Syst Rev 2008, CD000011.

15. Cramer JA: Enhancing patient compliance in the elderly. Role of packaging aids and monitoring. Drugs Aging 1998, 12:7-15.

16. Gould ON, Todd L, Irvine-Meek J: Adherence devices in a community sample: how are pillboxes used? Can Pharm J 2009, 142:28-35.
17. Hugtenburg JG, Timmers L, Elders PJ, Vervloet M, van Dijk L: Definitions, variants, and causes of nonadherence with medication: a challenge for tailored interventions. Patient Prefer Adherence 2013, 7:675-682.

18. Mahtani KR, Heneghan CJ, Glasziou PP, Perera R: Reminder packaging for improving adherence to self-administered long-term medications. Cochrane Database Syst Rev 2011, CD005025

19. Connor J, Rafter N, Rodgers A: Do fixed-dose combination pills or unit-of-use packaging improve adherence? A systematic review. Bull World Health Organ 2004, 82:935-939.

20. Zedler BK, Kakad P, Colilla S, Murrelle L, Shah NR: Does packaging with a calendar feature improve adherence to self-administered medication for long-term use? A systematic review. Clin Ther 2011, 33:62-73.

21. Bragge P, Clavisi O, Turner T, Tavender E, Collie A, Gruen RL: The Global Evidence Mapping Initiative: scoping research in broad topic areas. BMC Med Res Methodol 2011, 11:92

22. Schmucker C, Motschall E, Antes G, Meerpohl Jj: Methods of evidence mapping: a systematic review. Bundesgesundheitsblatt Gesundheitsforschung Gesundheitsschutz 2013, 56:1390-1397.

23. Hersberger KE, Boeni F, Arnet I: Dose-dispensing service as an intervention to improve adherence to polymedication. Expert Rev Clin Pharmacol 2013, 6:413-421.

24. Qualitative assessment tool for quantitative studies. [http://www.ephpp. $\mathrm{ca} /$ tools.html]

25. Thomas BH, Ciliska D, Dobbins M, Micucci S: A process for systematically reviewing the literature: providing the research evidence for public health nursing interventions. Worldviews Evid Based Nurs 2004, 1:176-184.

26. Armijo-Olivo S, Stiles CR, Hagen NA, Biondo PD, Cummings GG: Assessment of study quality for systematic reviews: a comparison of the Cochrane Collaboration Risk of Bias Tool and the Effective Public Health Practice Project Quality Assessment Tool: methodological research. J Eval Clin Pract 2012, 18:12-18.

27. Boutron I, Moher D, Altman DG, Schulz KF, Ravaud P: Extending the CONSORT statement to randomized trials of nonpharmacologic treatment: explanation and elaboration. Ann Intern Med 2008, 148:295-309.

28. The Cochrane Handbook for systematic reviews of interventions. [http://handbook.cochrane.org/]

29. Kozma CM, Reeder CE, Schulz RM: Economic, clinical, and humanistic outcomes: a planning model for pharmacoeconomic research. Clin Ther 1993, 15:1121-1132.

30. Ascione FJ, Shimp LA: The effectiveness of four education strategies in the elderly. Drug Intell Clin Pharm 1984, 18:926-931.

31. Azrin $\mathrm{NH}$, Teichner $\mathrm{G}$ : Evaluation of an instructional program for improving medication compliance for chronically mentally ill outpatients. Behav Res Ther 1998, 36:849-861.

32. Becker LA, Glanz K, Sobel E, Mossey J, Zinn S, Andrews Knott K: A randomized trial of special packaging of antihypertensive medications. J Fam Pract 1985, 22:357-361.

33. Binstock ML, Franklin $\mathrm{KL}$ : A comparison of compliance techniques on the control of high blood pressure. Am J Hypertens 1988, 1:192S-194S.

34. Crome $P$, Akehurst $M$, Keet J: Drug compliance in elderly hospital in-patients. Trial of the Dosett box. Practitioner 1980, 224:782-785.

35. Crome P, Curl B, Boswell M, Corless D, Lewis RR: Assessment of a new calendar pack - the 'C-Pak'. Age Ageing 1982, 11:275-279.

36. Eshelman FN, Fitzloff J: Effect of packaging on patient compliance with an antihypertensive medication. Curr Ther Res Clin Exp 1976, 20:215-219.

37. Fairley CK, Levy R, Rayner CR, Allardice K, Costello K, Thomas C, McArthur C, Kong D, Mijch A, Melbourne Adherence Group: Randomized trial of an adherence programme for clients with HIV. Int J STD AIDS 2003, 14:805-809.

38. Henry A, Batey RG: Enhancing compliance not a prerequisite for effective eradication of Helicobacter pylori: the HelP Study. Am J Gastroenterol 1999, 94:811-815.

39. Huang H-Y, Maguire MG, Miller ER III, Appel LJ: Impact of pill organizers and blister packs on adherence to pill taking in two vitamin supplementation trials. Am J Epidemio/ 2000, 152:780-787.

40. Lee JK, Grace KA, Taylor AJ: Effect of a pharmacy care program on medication adherence and persistence, blood pressure, and low-density lipoprotein cholesterol: a randomized controlled trial. JAMA 2006, 296:2563-2571. 
41. Lee M, Kemp JA, Canning A, Egan C, Tataronis G, Farraye FA: A randomized controlled trial of an enhanced patient compliance program for Helicobacter pylori therapy. Arch Intern Med 1999, 159:2312-2316.

42. MacDonald ET, MacDonald JB, Phoenix M: Improving drug compliance after hospital discharge. Br Med J 1977, 2:618-621.

43. Maier C, Mustapic D, Schuster E, Luger A, Eher R: Effect of a pocket-size tablet-dispensing device on glycaemic control in Type 2 diabetic patients. Diabet Med 2006, 23:40-45.

44. McPherson-Baker S, Malow RM, Penedo F, Jones DL, Schneiderman N, Klimas NG: Enhancing adherence to combination antiretroviral therapy in non-adherent HIV-positive men. AIDS Care 2000, 12:399-404.

45. Miaskowski C, Dodd M, West C, Schumacher K, Paul SM, Tripathy D, Koo P: Randomized clinical trial of the effectiveness of a self-care intervention to improve cancer pain management. J Clin Oncol 2004, 22:1713-1720.

46. Murray MD, Birt JA, Manatunga AK, Darnell JC: Medication compliance in elderly outpatients using twice-daily dosing and unit-of-use packaging Ann Pharmacother 1993, 27:616-621.

47. Nochowitz B, Shapiro NL, Nutescu EA, Cavallari LH: Effect of a warfarin adherence aid on anticoagulation control in an inner-city anticoagulation clinic population. Ann Pharmacother 2009, 43:1165-1172.

48. Park DC, Morrell RW, Frieske D, Kincaid D: Medication adherence behaviors in older adults: effects of external cognitive supports. Psychol Aging 1992, 7:252-256.

49. Peterson GM, McLean S, Millingen KS: A randomised trial of strategies to improve patient compliance with anticonvulsant therapy. Epilepsia 1984 25:412-417

50. Rheder TL, McCoy LK, Blackwell B, Whitehead W, Robinson A: Improving medication compliance by counseling and special prescription container. Am J Hosp Pharm 1980, 37:378-385

51. Schneider PJ, Murphy JE, Pedersen CA: Impact of medication packaging on adherence and treatment outcomes in older ambulatory patients. J Am Pharm Assoc 2008, 48:58-63.

52. Simmons D, Upjohn M, Gamble GD: Can medication packaging improve glycemic control and blood pressure in type 2 diabetes? Results from a randomized controlled trial. Diabetes Care 2000, 23:153-156

53. Skaer TL, Sclar DA, Markowski DJ, Won JK: Effect of value-added utilities on prescription refill compliance and Medicaid health care expenditures - a study of patients with non-insulin-dependent diabetes mellitus. J Clin Pharm Ther 1993, 18:295-299.

54. Skaer TL, Sclar DA, Markowski DJ, Won JK: Effect of value-added utilities on prescription refill compliance and health care expenditures for hypertension. J Hum Hypertens 1993, 7:515-518.

55. Solomon MZ, DeJong W: The impact of a clinic-based educational videotape on knowledge and treatment behavior of men with gonorrhea. Sex Transm Dis 1988, 15:127-132.

56. Valenstein M, Kavanagh J, Lee T, Reilly P, Dalack GW, Grabowski J, Smelson D, Ronis DL, Ganoczy D, Woltmann E, Metreger T, Wolschon P, Jensen A, Poddig B, Blow FC: Using a pharmacy-based intervention to improve antipsychotic adherence among patients with serious mental illness. Schizophr Bull 2009, 37:727-736.

57. Ware GJ, Holford NH, Davison JG, Harris RG: Unit dose calendar packaging and elderly patient compliance. N Z Med J 1991, 104:495-497.

58. Winland-Brown JE, Valiante J: Effectiveness of different medication management approaches on elders' medication adherence. Outcomes Manag Nurs Pract 2000, 4:172-176.

59. Begg C, Cho M, Eastwood S, Horton R, Moher D, Olkin I, Pitkin R, Rennie D, Schulz KF, Simel D, Stroup DF: Improving the quality of reporting of randomized controlled trials. The CONSORT statement. JAMA 1996, 276:637-639.

60. Haynes RB, McKibbon KA, Kanani R: Systematic review of randomised trials of interventions to assist patients to follow prescriptions for medications. Lancet 1996, 348:383-386.

61. Chan A-W, Altman DG: Epidemiology and reporting of randomised trials published in PubMed journals. Lancet 2005, 365:1159-1162.

62. Hopewell S, Dutton S, Yu L-M, Chan A-W, Altman DG: The quality of reports of randomised trials in 2000 and 2006: comparative study of articles indexed in PubMed. BMJ 2010, 340.

63. Turner L, Shamseer L, Altman DG, Weeks L, Peters J, Kober T, Dias S, Schulz KF, Plint AC, Moher D: Consolidated standards of reporting trials (CONSORT) and the completeness of reporting of randomised controlled trials (RCTs) published in medical journals. Cochrane Database Syst Rev 2012, MR000030.

64. Huwiler-Muntener K, Juni P, Junker C, Egger M: Quality of reporting of randomized trials as a measure of methodologic quality. JAMA 2002, 287:2801-2804.

65. Glasziou P, Meats $E$, Heneghan C, Shepperd S: What is missing from descriptions of treatment in trials and reviews? BMJ 2008, 336:1472-1474.

66. Dopson S, Locock L, Chambers D, Gabbay J: Implementation of evidencebased medicine: evaluation of the Promoting Action on Clinical Effectiveness programme. J Health Serv Res Policy 2001, 6:23-31.

67. Ostrop NJ, Gill MJ: Antiretroviral medication adherence and persistence with respect to adherence tool usage. AIDS Patient Care STDS 2000, 14:351-358.

68. Petersen ML, Wang Y, van der Laan MJ, Guzman D, Riley E, Bangsberg DR: Pillbox organizers are associated with improved adherence to HIV antiretroviral therapy and viral suppression: a marginal structural model analysis. Clin Infect Dis 2007, 45:908-915.

69. Wilson S, Delaney BC, Roalfe A, Roberts L, Redman V, Wearn AM, Hobbs FD: Randomised controlled trials in primary care: case study. BMJ 2000, 321:24-27.

70. Moser M: Randomized clinical trials: alternatives to conventional randomization. Am J Emerg Med 1986, 4:276-285.

71. Atkin PA, Finnegan TP, Ogle SJ, Shenfield GM: Functional ability of patients to manage medication packaging: a survey of geriatric inpatients. Age Ageing 1994, 23:113-116

72. Rivers PH: Compliance aids - do they work? Drugs Aging 1992, 2:103-111.

73. Levings B, Szep S, Helps S: Towards the safer use of dosettes. J Qual Clin Pract 1999, 19:69-72

74. Nunney J, Raynor DK: Mind the gap: how compliance aids increase distance between patients and their medicines. Int J Pharm Pract 2001, 9(suppl):R46.

doi:10.1186/2046-4053-3-29

Cite this article as: Boeni et al:: Effect of drug reminder packaging on medication adherence: a systematic review revealing research gaps. Systematic Reviews 2014 3:29.

\section{Submit your next manuscript to BioMed Central and take full advantage of:}

- Convenient online submission

- Thorough peer review

- No space constraints or color figure charges

- Immediate publication on acceptance

- Inclusion in PubMed, CAS, Scopus and Google Scholar

- Research which is freely available for redistribution 